Supplement of Hydrol. Earth Syst. Sci., 25, 4789-4805, 2021

https://doi.org/10.5194/hess-25-4789-2021-supplement

(C) Author(s) 2021. CC BY 4.0 License.

(c) (i)

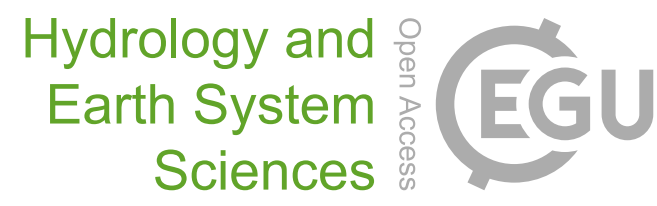

Supplement of

\title{
Applicability of Landsat 8 thermal infrared sensor for identifying submarine groundwater discharge springs in the Mediterranean Sea basin
}

Sònia Jou-Claus et al.

Correspondence to: Sònia Jou-Claus (soniajouclaus@gmail.com)

The copyright of individual parts of the supplement might differ from the article licence. 
Supplementary material 1

\begin{tabular}{|c|c|c|c|c|c|c|c|}
\hline Country & \multicolumn{2}{|c|}{ ID/Name } & \multirow{2}{*}{\begin{tabular}{|l}
$\begin{array}{l}\text { Flow } \\
\text { rate } \\
\left(\mathbf{m} \cdot \mathbf{S}^{-1}\right)\end{array}$ \\
0.031
\end{tabular}} & \multirow{2}{*}{$\begin{array}{c}\text { Depth (m) } \\
15-20\end{array}$} & \multirow{2}{*}{$\begin{array}{c}\begin{array}{c}\text { Distance } \\
(\mathbf{m})\end{array} \\
0 \text { (on the } \\
\text { cliff side) }\end{array}$} & \multirow{2}{*}{$\begin{array}{l}\text { Type } \\
\text { ND }\end{array}$} & \multirow{2}{*}{$\begin{array}{c}\text { Literature } \\
\text { Backalowicz, } 2018\end{array}$} \\
\hline \multirow{15}{*}{ Spain } & 1 & $\begin{array}{l}\text { Maro-Cerro } \\
\text { Gordo }\end{array}$ & & & & & \\
\hline & 2 & Moraig & $0.3-9.0$ & -12 & $\begin{array}{l}0 \text { (on the } \\
\text { cliff side) }\end{array}$ & 2 & Fleury et al., 2007 \\
\hline & 3 & Toix & ND & -8 & Seashore & 2 & Fleury et al., 2007 \\
\hline & 4 & Agua dulce & ND & $\begin{array}{c}\text { Coastal } \\
\text { and } \\
\text { submarine }\end{array}$ & ND & ND & Backalowicz, 2018 \\
\hline & 5 & $\begin{array}{l}\text { Almirante } \\
\text { Gallinera }\end{array}$ & ND & ND & ND & ND & Backalowicz, 2018 \\
\hline & 6 & $\begin{array}{c}\text { Alfaro- } \\
\text { Mediodia } \\
\end{array}$ & $\begin{array}{c}65 \text { and } \\
84 \mathrm{hm} 3 / \mathrm{y}\end{array}$ & ND & ND & ND & Backalowicz, 2018 \\
\hline & 7 & $\begin{array}{c}\text { Torre } \\
\text { Badum }\end{array}$ & 2 & 0 & $<10$ & ND & Mejías et al., 2012 \\
\hline & 8 & La Fuentes & $0.03-0.17$ & ND & $<10$ & ND & Mejías et al., 2012 \\
\hline & 9 & Font de Dins & $1.1-0.4$ & ND & $<10$ & ND & Mejías et al., 2012 \\
\hline & 10 & La Falconera & 0.1 & ND & 0 & ND & Backalowicz, 2018 \\
\hline & 11 & Alcaufar & ND & $\mathrm{ND}$ & $\mathrm{ND}$ & $\mathrm{ND}$ & $\begin{array}{c}\text { Garcia-Solsona et } \\
\text { al., } 2010\end{array}$ \\
\hline & 12 & $\begin{array}{l}\text { Migjorn-El } \\
\text { Pla }\end{array}$ & 1 & ND & ND & ND & $\begin{array}{c}\text { Basterretxea et al., } \\
2010\end{array}$ \\
\hline & 13 & Llevant & 0.009 & ND & ND & ND & $\begin{array}{c}\text { Basterretxea et al., } \\
2010\end{array}$ \\
\hline & 14 & $\begin{array}{l}\text { Font des } \\
\text { Verger } \\
\end{array}$ & $0.01-1$ & ND & ND & ND & $\begin{array}{c}\text { Basterretxea et al., } \\
2010 \\
\end{array}$ \\
\hline & 15 & Ses Ufanes & $20-30$ & ND & ND & ND & $\begin{array}{c}\text { Basterretxea et al., } \\
2010\end{array}$ \\
\hline \multirow{6}{*}{ France } & 16 & Fitou & 0.1 & ND & ND & 2 & $\begin{array}{c}\text { Bejannin et al., } \\
2017\end{array}$ \\
\hline & 17 & Laucate & ND & ND & ND & 2 & $\begin{array}{c}\text { Bejannin et al., } \\
2017\end{array}$ \\
\hline & 18 & $\begin{array}{c}\text { Fontdame- } \\
\text { Fontestramar }\end{array}$ & $1-15$ & 4 & 100 & 2 & Fleury et al., 2007 \\
\hline & 19 & Vise & $>0.1$ & -30 & ND & 3 & $\begin{array}{c}\text { Bejannin et al., } \\
2017\end{array}$ \\
\hline & 20 & Port-Miou & $0.5-0.7$ & -12 & ND & 2 & Fleury et al., 2007 \\
\hline & 21 & Bestoua & ND & ND & ND & 2 & Fleury et al., 2007 \\
\hline \multirow{7}{*}{ Italy } & 22 & Mortola & 0.2 & -36 & 800 & 1 & Fleury et al., 2007 \\
\hline & 23 & $\begin{array}{c}\text { Bue Marino } \\
\text { Cave } \\
\text { (Sardinia) }\end{array}$ & ND & ND & ND & ND & Backalowicz, 2018 \\
\hline & 24 & Taranto & ND & -19 & ND & ND & Fleury et al., 2007 \\
\hline & 25 & Cefalú & $\mathrm{ND}$ & ND & ND & ND & Backalowicz, 2018 \\
\hline & 26 & Castellamare & $\mathrm{ND}$ & ND & ND & ND & Backalowicz, 2018 \\
\hline & 27 & Donnalucata & $\begin{array}{c}0.004- \\
0.012 \\
\end{array}$ & ND & $\begin{array}{l}\text { Onshore } \\
\text { and } \\
\text { offshore }\end{array}$ & 1 & $\begin{array}{c}\text { Burnett\&Dulaiova } \\
2006\end{array}$ \\
\hline & 28 & Pozzalo & $\mathrm{ND}$ & ND & ND & ND & Backalowicz, 2018 \\
\hline \multirow{5}{*}{ Croatia } & 29 & South Istria & 0.024 & ND & ND & ND & Backalowicz, 2018 \\
\hline & 30 & Martinscica & 0.4 & ND & ND & ND & Backalowicz, 2018 \\
\hline & 31 & Perilo & $\mathrm{ND}$ & ND & ND & ND & Backalowicz, 2018 \\
\hline & 32 & Novijanska & $>0.4$ & ND & ND & ND & Backalowicz, 2018 \\
\hline & 33 & Jurjevska & $50-0.6$ & ND & ND & ND & Backalowicz, 2018 \\
\hline
\end{tabular}




\begin{tabular}{|c|c|c|c|c|c|c|c|}
\hline & 34 & Patan & $0.5-10$ & 2.7 & 500 & ND & Backalowicz, 2018 \\
\hline & 35 & Slano & ND & ND & ND & ND & Backalowicz, 2018 \\
\hline & 36 & Robinzon & 2 & ND & $\mathrm{ND}$ & ND & Backalowicz, 2018 \\
\hline \multirow{6}{*}{ Grece } & 37 & Cephalonia & $1-10$ & 0 & Seashore & 2 & Fleury et al., 2007 \\
\hline & 38 & $\begin{array}{c}\text { Kiveri- } \\
\text { Anavalos } \\
\end{array}$ & $9-11$ & 0 to -7 & $\begin{array}{c}0 \text { to few } \\
\mathrm{m}\end{array}$ & 3 & Fleury et al., 2007 \\
\hline & 39 & E.argolis & ND & ND & ND & ND & Backalowicz, 2018 \\
\hline & 40 & Parnon & ND & ND & ND & ND & Backalowicz, 2018 \\
\hline & 41 & $\begin{array}{c}\text { Almiros of } \\
\text { Iraklio }\end{array}$ & $2-30$ & 3 & 1000 & 2 & Fleury et al., 2007 \\
\hline & 42 & $\begin{array}{c}\text { Agios } \\
\text { Nikolaos }\end{array}$ & $>2.3$ & $4-7$ & 0.5 & 2 & Fleury et al., 2007 \\
\hline \multirow{8}{*}{ Turkey } & 43 & Gokova-1 & $0.02-0.10$ & 0 & Seashore & 1 & $\begin{array}{l}\text { Bayari\&Kurttas., } \\
2015\end{array}$ \\
\hline & 44 & Gokova-2 & ND & 0 & Seashore & 1 & $\begin{array}{c}\text { Bayari\&Kurttas., } \\
2015 \\
\end{array}$ \\
\hline & 45 & Gokova-3 & ND & 0 & Seashore & 1 & $\begin{array}{c}\text { Bayari\&Kurttas., } \\
2015\end{array}$ \\
\hline & 46 & Antalya-1 & $\mathrm{ND}$ & $\mathrm{ND}$ & ND & ND & Backalowicz, 2018 \\
\hline & 47 & Antalya-2 & ND & ND & ND & ND & Backalowicz, 2018 \\
\hline & 48 & Antalya-3 & ND & ND & ND & ND & Backalowicz, 2018 \\
\hline & 49 & $\begin{array}{c}\text { Lefke } \\
\text { (Chipre) }\end{array}$ & ND & ND & ND & ND & Backalowicz, 2018 \\
\hline & 50 & Ovacik & 0.74 & -1.5 to -3 & $2 \mathrm{~m}$ & 1 & Elhatip, 2003 \\
\hline Syria & 51 & Latakia & & & & & Backalowicz, 2018 \\
\hline Lebanon & 52 & Chekka & 60 & $\begin{array}{l}-10 \text { to }- \\
150\end{array}$ & $\begin{array}{c}100 \mathrm{~m}- \\
1 \mathrm{~km}\end{array}$ & 2 & Fleury et al., 2007 \\
\hline Israel & 53 & Dor & $4.05 * 10^{-11}$ & ND & ND & ND & $\begin{array}{c}\text { Weinstein et al., } \\
2011\end{array}$ \\
\hline Libya & 54 & Ain Zayanah & $1-5$ & $0-1$ & Seashore & 2 & Fleury et al., 2007 \\
\hline
\end{tabular}




\section{Supplementary material 2}

\begin{tabular}{|c|c|c|c|c|c|c|}
\hline Site & ID & Date & Time & Path & Row & $\begin{array}{c}\text { Scene } \\
\text { cloud } \\
\%\end{array}$ \\
\hline \multirow{19}{*}{1} & LC08_L1TP_200035_20181231_20190130_01_T1 & $31 / 12 / 18$ & $10: 50$ & 200 & 35 & 4.91 \\
\hline & LC08_L1TP_200035_20181215_20181227_01_T1 & $15 / 12 / 18$ & $10: 50$ & 200 & 35 & 0.23 \\
\hline & LC08_L1TP_200035_20181012_20181030_01_T1 & $12 / 10 / 18$ & $10: 50$ & 200 & 35 & 0.02 \\
\hline & LC08_L1TP_200035_20180926_20181009_01_T1 & $26 / 9 / 18$ & $10: 50$ & 200 & 35 & 10.02 \\
\hline & LC08_L1TP_200035_20180825_20180829_01_T1 & $25 / 8 / 18$ & $10: 50$ & 200 & 35 & 9.44 \\
\hline & LC08_L1TP_200035_20180809_20180815_01_T1 & $9 / 8 / 18$ & $10: 50$ & 200 & 35 & 0.54 \\
\hline & LC08_L1TP_200035_20180708_20180717_01_T1 & $8 / 7 / 18$ & $10: 50$ & 200 & 35 & 14.06 \\
\hline & LC08_L1TP_200035_20180622_20180703_01_T1 & $22 / 6 / 18$ & $10: 50$ & 200 & 35 & 23.21 \\
\hline & LC08_L1TP_200035_20180419_20180501_01_T1 & $19 / 4 / 18$ & $10: 50$ & 200 & 35 & 14.13 \\
\hline & LC08_L1TP_200035_20180403_20180417_01_T1 & $3 / 4 / 18$ & $10: 50$ & 200 & 35 & 5.02 \\
\hline & LC08_L1TP_200035_20171212_20171223_01_T1 & $12 / 12 / 17$ & $10: 50$ & 200 & 35 & 4.01 \\
\hline & LC08_L1TP_200035_20171110_20171121_01_T1 & $10 / 11 / 17$ & $10: 50$ & 200 & 35 & 14.10 \\
\hline & LC08_L1TP_200035_20171025_20171107_01_T1 & $25 / 10 / 17$ & $10: 50$ & 200 & 35 & 10.81 \\
\hline & LC08_L1TP_200035_20170923_20171013_01_T1 & $23 / 9 / 17$ & 10:50 & 200 & 35 & 42.87 \\
\hline & LC08_L1TP_200035_20170822_20170911_01_T1 & $22 / 8 / 17$ & $10: 50$ & 200 & 35 & 3.56 \\
\hline & LC08_L1TP_200035_20170721_20170728_01_T1 & $21 / 7 / 17$ & $10: 50$ & 200 & 35 & 0.07 \\
\hline & LC08_L1TP_200035_20170518_20170525_01_T1 & $18 / 5 / 17$ & $10: 50$ & 200 & 35 & 1.92 \\
\hline & LC08_L1TP_200035_20170331_20170414_01_T1 & $31 / 3 / 17$ & $10: 50$ & 200 & 35 & 11.58 \\
\hline & LC08_L1TP_200035_20170315_20170328_01_T1 & $15 / 3 / 17$ & $10: 50$ & 200 & 35 & 57.47 \\
\hline \multirow{20}{*}{$2,3,4,5,6$} & LC08_L1TP_198033_20181217_20181227_01_T1 & $17 / 12 / 18$ & $10: 37$ & 198 & 33 & 11,03 \\
\hline & LC08_L1TP_198033_20181201_20181211_01_T1 & $1 / 12 / 18$ & $10: 37$ & 198 & 33 & 34,05 \\
\hline & LC08_L1TP_198033_20180928_20181009_01_T1 & $28 / 10 / 18$ & $10: 37$ & 198 & 33 & 6,42 \\
\hline & LC08_L1TP_198033_20180827_20180911_01_T1 & $27 / 9 / 18$ & $10: 37$ & 198 & 33 & 6,18 \\
\hline & LC08_L1TP_198033_20180726_20180731_01_T1 & $26 / 7 / 18$ & $10: 37$ & 198 & 33 & 1,52 \\
\hline & LC08_L1TP_198033_20180710_20180717_01_T1 & $10 / 7 / 18$ & $10: 37$ & 198 & 33 & 1,15 \\
\hline & LC08_L1TP_198033_20180624_20180704_01_T1 & $24 / 6 / 18$ & $10: 37$ & 198 & 33 & 6,18 \\
\hline & LC08_L1TP_198033_20180405_20180417_01_T1 & $5 / 4 / 18$ & $10: 37$ & 198 & 33 & 0,2 \\
\hline & LC08_L1TP_198033_20180131_20180207_01_T1 & $31 / 1 / 18$ & $10: 37$ & 198 & 33 & 5,35 \\
\hline & LC08_L1TP_198033_20171230_20180103_01_T1 & $30 / 12 / 17$ & $10: 37$ & 198 & 33 & 0,7 \\
\hline & LC08_L1TP_198033_20171112_20171121_01_T1 & $12 / 11 / 17$ & $10: 37$ & 198 & 33 & 0,14 \\
\hline & LC08_L1TP_198033_20171027_20171107_01_T1 & $27 / 10 / 17$ & $10: 37$ & 198 & 33 & 0,07 \\
\hline & LC08_L1TP_198033_20171011_20171024_01_T1 & $11 / 10 / 17$ & $10: 37$ & 198 & 33 & 6,68 \\
\hline & LC08_L1TP_198033_20170925_20171013_01_T1 & $25 / 9 / 17$ & $10: 37$ & 198 & 33 & 0,31 \\
\hline & LC08_L1TP_198033_20170824_20170913_01_T1 & $24 / 8 / 17$ & $10: 37$ & 198 & 33 & 3,99 \\
\hline & LC08_L1TP_198033_20170621_20170630_01_T1 & $21 / 6 / 17$ & $10: 37$ & 198 & 33 & 5,76 \\
\hline & LC08_L1TP_198033_20170520_20170525_01_T1 & $20 / 5 / 17$ & $10: 37$ & 198 & 33 & 0,28 \\
\hline & LC08_L1TP_198033_20170504_20170515_01_T1 & $4 / 5 / 17$ & $10: 37$ & 198 & 33 & 8,82 \\
\hline & LC08_L1TP_198033_20170402_20170414_01_T1 & $2 / 4 / 17$ & $10: 37$ & 198 & 33 & 0,09 \\
\hline & LC08_L1TP_198033_20170301_20170316_01_T1 & $1 / 3 / 17$ & $10: 37$ & 198 & 33 & 2,78 \\
\hline
\end{tabular}




\begin{tabular}{|c|c|c|c|c|c|c|}
\hline & LC08_L1TP_198033_20170112_20170311_01_T1 & $12 / 1 / 17$ & $10: 37$ & 198 & 33 & 1,13 \\
\hline \multirow{23}{*}{$7,8,9$} & LC08_L1TP_198032_20180928_20181009_01_T1 & 28/9/18 & $10: 36$ & 198 & 32 & 0,42 \\
\hline & LC08_L1TP_198032_20180726_20180731_01_T1 & $26 / 7 / 18$ & $10: 36$ & 198 & 32 & 0,28 \\
\hline & LC08_L1TP_198032_20180710_20180717_01_T1 & $10 / 7 / 18$ & $10: 36$ & 198 & 32 & 2,36 \\
\hline & LC08_L1TP_198032_20180624_20180704_01_T1 & $24 / 6 / 18$ & $10: 36$ & 198 & 32 & 19,94 \\
\hline & LC08_L1TP_198032_20180421_20180502_01_T1 & $21 / 4 / 18$ & $10: 36$ & 198 & 32 & 16,3 \\
\hline & LC08_L1TP_198032_20180405_20180417_01_T1 & $5 / 4 / 18$ & $10: 36$ & 198 & 32 & 0,07 \\
\hline & LC08_L1TP_198032_20180131_20180207_01_T1 & $31 / 1 / 18$ & $10: 36$ & 198 & 32 & 8,41 \\
\hline & LC08_L1TP_198032_20180115_20180120_01_T1 & $15 / 1 / 18$ & $10: 36$ & 198 & 32 & 10,19 \\
\hline & LC08_L1TP_198032_20171230_20180103_01_T1 & $30 / 12 / 17$ & $10: 36$ & 198 & 32 & 14,49 \\
\hline & LC08_L1TP_198032_20171128_20171207_01_T1 & $28 / 11 / 17$ & $10: 36$ & 198 & 32 & 0,81 \\
\hline & LC08_L1TP_198032_20171112_20171121_01_T1 & $12 / 11 / 17$ & $10: 36$ & 198 & 32 & 0,75 \\
\hline & LC08_L1TP_198032_20171027_20171107_01_T1 & $27 / 10 / 17$ & $10: 36$ & 198 & 32 & 0,27 \\
\hline & LC08_L1TP_198032_20170925_20171013_01_T1 & $25 / 9 / 17$ & $10: 36$ & 198 & 32 & 11,41 \\
\hline & LC08_L1TP_198032_20170824_20170913_01_T1 & $24 / 8 / 17$ & $10: 36$ & 198 & 32 & 3,36 \\
\hline & LC08_L1TP_198032_20170808_20170823_01_T1 & $8 / 8 / 17$ & $10: 36$ & 198 & 32 & 24,18 \\
\hline & LC08_L1TP_198032_20170621_20170630_01_T1 & $21 / 6 / 17$ & $10: 36$ & 198 & 32 & 0,27 \\
\hline & LC08_L1TP_198032_20170520_20170525_01_T1 & $20 / 5 / 17$ & $10: 36$ & 198 & 32 & 3,16 \\
\hline & LC08_L1TP_198032_20170504_20170515_01_T1 & $4 / 5 / 17$ & $10: 36$ & 198 & 32 & 9,31 \\
\hline & LC08_L1TP_198032_20170418_20170501_01_T1 & $18 / 4 / 17$ & $10: 36$ & 198 & 32 & 4,92 \\
\hline & LC08_L1TP_198032_20170402_20170414_01_T1 & $2 / 4 / 17$ & $10: 36$ & 198 & 32 & 7,42 \\
\hline & LC08_L1TP_198032_20170317_20170328_01_T1 & $17 / 3 / 17$ & $10: 36$ & 198 & 32 & 0,13 \\
\hline & LC08_L1TP_198032_20170301_20170316_01_T1 & $1 / 3 / 17$ & $10: 36$ & 198 & 32 & 0,03 \\
\hline & LC08_L1TP_198032_20170112_20170311_01_T1 & $12 / 1 / 17$ & $10: 36$ & 198 & 32 & 4,83 \\
\hline \multirow{18}{*}{10} & LC08_L1TP_197031_20181124_20181210_01_T1 & $24 / 11 / 18$ & $10: 30$ & 197 & 31 & 8,24 \\
\hline & LC08_L1TP_197031_20181023_20181031_01_T1 & $23 / 10 / 18$ & $10: 30$ & 197 & 31 & 2,27 \\
\hline & LC08_L1TP_197031_20180921_20180928_01_T1 & $21 / 9 / 18$ & $10: 30$ & 197 & 31 & 5,19 \\
\hline & LC08_L1TP_197031_20180820_20180829_01_T1 & $20 / 8 / 18$ & $10: 30$ & 197 & 31 & 0,4 \\
\hline & LC08_L1TP_197031_20180804_20180814_01_T1 & $4 / 8 / 18$ & $10: 30$ & 197 & 31 & 11,54 \\
\hline & LC08_L1TP_197031_20180719_20180731_01_T1 & $19 / 7 / 18$ & $10: 30$ & 197 & 31 & 1,32 \\
\hline & LC08_L1TP_197031_20180703_20180717_01_T1 & $3 / 7 / 18$ & $10: 30$ & 197 & 31 & 10,04 \\
\hline & LC08_L1TP_197031_20180601_20180614_01_T1 & $1 / 6 / 18$ & $10: 30$ & 197 & 31 & 17,96 \\
\hline & LC08_L1TP_197031_20180430_20180516_01_T1 & $30 / 4 / 18$ & $10: 30$ & 197 & 31 & 21,24 \\
\hline & LC08_L1TP_197031_20180209_20180222_01_T1 & $9 / 2 / 18$ & $10: 30$ & 197 & 31 & 2,4 \\
\hline & LC08_L1TP_197031_20180124_20180206_01_T1 & $24 / 1 / 18$ & $10: 30$ & 197 & 31 & 0,91 \\
\hline & LC08_L1TP_197031_20171223_20180103_01_T1 & $23 / 12 / 17$ & $10: 30$ & 197 & 31 & 6,88 \\
\hline & LC08_L1TP_197031_20171121_20171206_01_T1 & $21 / 11 / 17$ & $10: 30$ & 197 & 31 & 0,54 \\
\hline & LC08_L1TP_197031_20170902_20170916_01_T1 & 2/9/17 & $10: 30$ & 197 & 31 & 11,32 \\
\hline & LC08_L1TP_197031_20170817_20170826_01_T1 & $17 / 8 / 17$ & $10: 30$ & 197 & 31 & 0,22 \\
\hline & LC08_L1TP_197031_20170716_20170727_01_T1 & $16 / 7 / 17$ & $10: 30$ & 197 & 31 & 4,06 \\
\hline & LC08_L1TP_197031_20170630_20170715_01_T1 & $30 / 6 / 17$ & $10: 30$ & 197 & 31 & 16,01 \\
\hline & LC08_L1TP_197031_20170614_20170628_01_T1 & $14 / 6 / 17$ & $10: 30$ & 197 & 31 & 0,66 \\
\hline
\end{tabular}




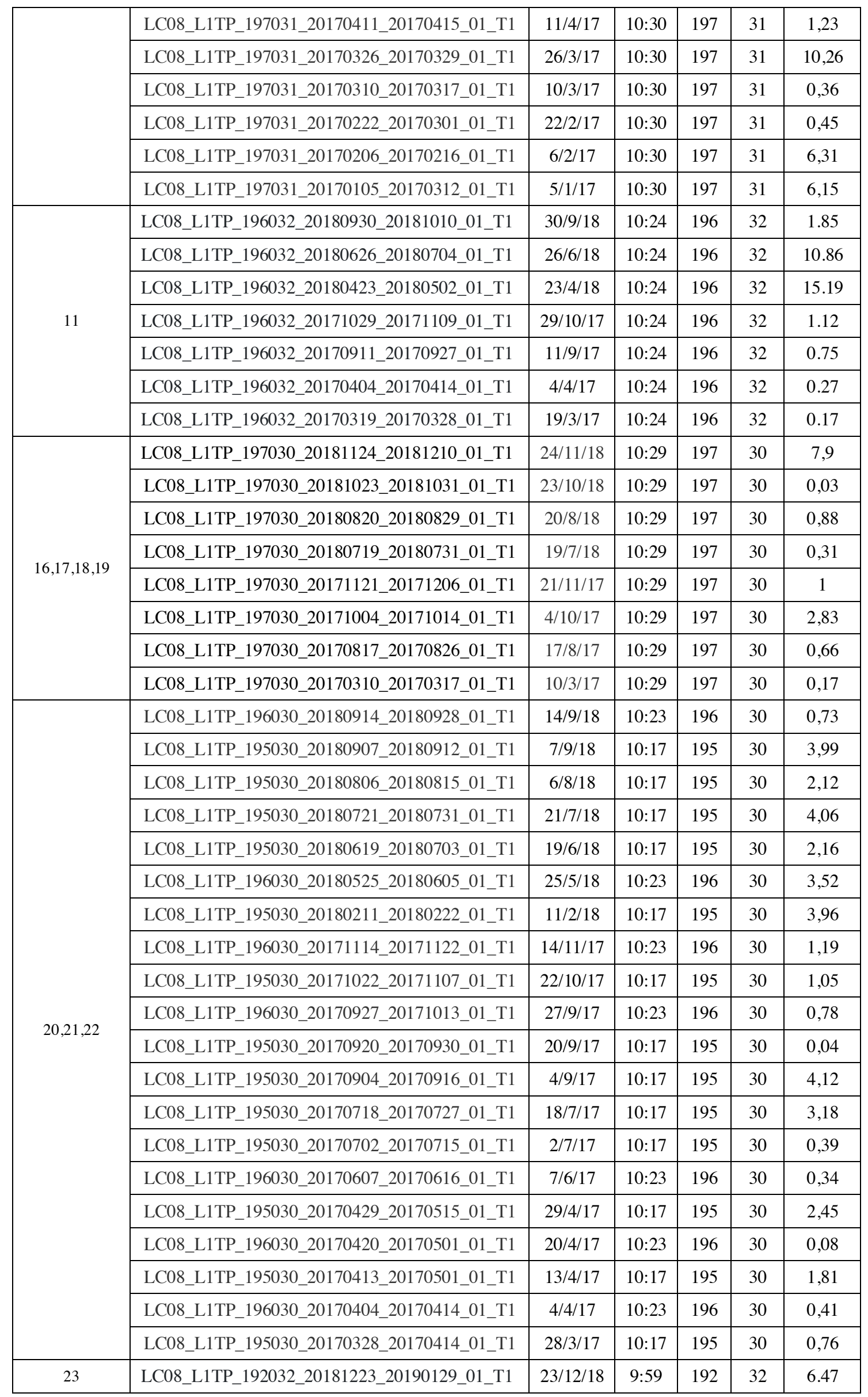




\begin{tabular}{|c|c|c|c|c|c|c|}
\hline & LC08_L1TP_192032_20181121_20181129_01_T2 & $21 / 11 / 18$ & 9:59 & 192 & 32 & 29.83 \\
\hline & LC08_L1TP_192032_20180817_20180829_01_T1 & $17 / 8 / 18$ & $9: 59$ & 192 & 32 & 2.46 \\
\hline & LC08_L1TP_192032_20180630_20180716_01_T1 & $30 / 6 / 18$ & 9:59 & 192 & 32 & 0.7 \\
\hline & LC08_L1TP_192032_20180105_20180118_01_T1 & $5 / 1 / 18$ & 9:59 & 192 & 32 & 14.46 \\
\hline & LC08_L1TP_192032_20171017_20171024_01_T1 & $17 / 10 / 17$ & 9:59 & 192 & 32 & 0.79 \\
\hline & LC08_L1TP_192032_20170830_20170914_01_T1 & $30 / 8 / 17$ & 9:59 & 192 & 32 & 0.01 \\
\hline & LC08_L1TP_192032_20170729_20170811_01_T1 & 29/7/17 & 9:59 & 192 & 32 & 0.40 \\
\hline & LC08_L1TP_192032_20170627_20170714_01_T1 & $27 / 6 / 17$ & 9:59 & 192 & 32 & 0.03 \\
\hline & LC08_L1TP_192032_20170408_20180523_01_T1 & $8 / 4 / 17$ & 9:59 & 192 & 32 & 0 \\
\hline & LC08_L1TP_192032_20170219_20180527_01_T1 & $19 / 2 / 17$ & 9:59 & 192 & 32 & 6.49 \\
\hline \multirow{9}{*}{24} & LC08_L1TP_188032_20181227_20190129_01_T1 & $27 / 12 / 18$ & 9.34 & 188 & 32 & 0.59 \\
\hline & LC08_L1TP_188032_20181109_20181116_01_T1 & $9 / 11 / 18$ & 9.34 & 188 & 32 & 1.02 \\
\hline & LC08_L1TP_188032_20180906_20180912_01_T1 & $6 / 9 / 18$ & 9.34 & 188 & 32 & 0 \\
\hline & LC08_L1TP_188032_20180805_20180814_01_T1 & $5 / 8 / 18$ & 9.34 & 188 & 32 & 8.21 \\
\hline & LC08_L1TP_188032_20180125_20180206_01_T1 & $25 / 1 / 18$ & 9.34 & 188 & 32 & 1.10 \\
\hline & LC08_L1TP_188032_20171224_20180103_01_T1 & $24 / 12 / 17$ & 9.34 & 188 & 32 & 0.86 \\
\hline & LC08_L1TP_188032_20170818_20170826_01_T1 & $8 / 8 / 17$ & 9.34 & 188 & 32 & 0.17 \\
\hline & LC08_L1TP_188032_20170412_20180527_01_T1 & $12 / 4 / 17$ & 9.34 & 188 & 32 & 5.32 \\
\hline & LC08_L1TP_188032_20170223_20170301_01_T1 & $23 / 2 / 17$ & 9.34 & 188 & 32 & 29.05 \\
\hline \multirow{11}{*}{$25,26,27,28$} & LC08_L1TP_189034_20180929_20181009_01_T1 & 29/9/18 & $9: 41$ & 189 & 34 & 1.50 \\
\hline & LC08_L1TP_189034_20180812_20180815_01_T1 & $12 / 8 / 18$ & $9: 41$ & 189 & 34 & 6.32 \\
\hline & LC08_L1TP_189034_20180727_20180731_01_T1 & $27 / 7 / 18$ & $9: 41$ & 189 & 34 & 4.69 \\
\hline & LC08_L1TP_189034_20180609_20180615_01_T1 & $9 / 6 / 18$ & $9: 41$ & 189 & 34 & 5.33 \\
\hline & LC08_L1TP_189034_20180524_20180605_01_T1 & $24 / 5 / 18$ & $9: 41$ & 189 & 34 & 3.62 \\
\hline & LC08_L1TP_189034_20180406_20180417_01_T1 & $6 / 4 / 18$ & $9: 41$ & 189 & 34 & 0.54 \\
\hline & LC08_L1TP_189034_20171215_20171224_01_T1 & $15 / 12 / 17$ & $9: 41$ & 189 & 34 & 2.97 \\
\hline & LC08_L1TP_189034_20171012_20171024_01_T1 & $12 / 10 / 17$ & $9: 41$ & 189 & 34 & 5.61 \\
\hline & LC08_L1TP_189034_20170825_20180527_01_T1 & $25 / 8 / 17$ & $9: 41$ & 189 & 34 & 0 \\
\hline & LC08_L1TP_189034_20170622_20170630_01_T1 & $22 / 6 / 17$ & $9: 41$ & 189 & 34 & 3.21 \\
\hline & LC08_L1TP_189034_20170318_20170328_01_T1 & $18 / 3 / 17$ & $9: 41$ & 189 & 34 & 0 \\
\hline \multirow{12}{*}{$12,13,14,15$} & LC08_L1TP_197032_20181023_20181031_01_T1 & $23 / 10 / 18$ & $10: 30$ & 197 & 32 & 2,22 \\
\hline & LC08_L1TP_196033_20180930_20181010_01_T1 & $30 / 9 / 18$ & $10: 24$ & 196 & 33 & 1,46 \\
\hline & LC08_L1TP_197032_20180921_20180928_01_T1 & $21 / 9 / 18$ & $10: 30$ & 197 & 32 & 1,16 \\
\hline & LC08_L1TP_196033_20180829_20180911_01_T1 & $29 / 8 / 18$ & $10: 24$ & 196 & 33 & 5,45 \\
\hline & LC08_L1TP_197032_20180820_20180829_01_T1 & $20 / 8 / 18$ & $10: 30$ & 197 & 32 & 0,33 \\
\hline & LC08_L1TP_197032_20180804_20180814_01_T1 & $4 / 8 / 18$ & $10: 30$ & 197 & 32 & 9,79 \\
\hline & LC08_L1TP_196033_20180728_20180731_01_T1 & $28 / 7 / 18$ & $10: 24$ & 196 & 33 & 7,18 \\
\hline & LC08_L1TP_197032_20180719_20180731_01_T1 & $19 / 7 / 18$ & $10: 30$ & 197 & 32 & 0,91 \\
\hline & LC08_L1TP_196033_20180712_20180717_01_T1 & $12 / 7 / 18$ & $10: 24$ & 196 & 33 & 1,99 \\
\hline & LC08_L1TP_197032_20180703_20180717_01_T1 & $3 / 7 / 18$ & $10: 30$ & 197 & 32 & 5,07 \\
\hline & LC08_L1TP_197032_20180617_20180703_01_T1 & $17 / 6 / 18$ & $10: 30$ & 197 & 32 & 15,65 \\
\hline & LC08_L1TP_197032_20180601_20180614_01_T1 & $1 / 6 / 18$ & $10: 30$ & 197 & 32 & 4,68 \\
\hline
\end{tabular}




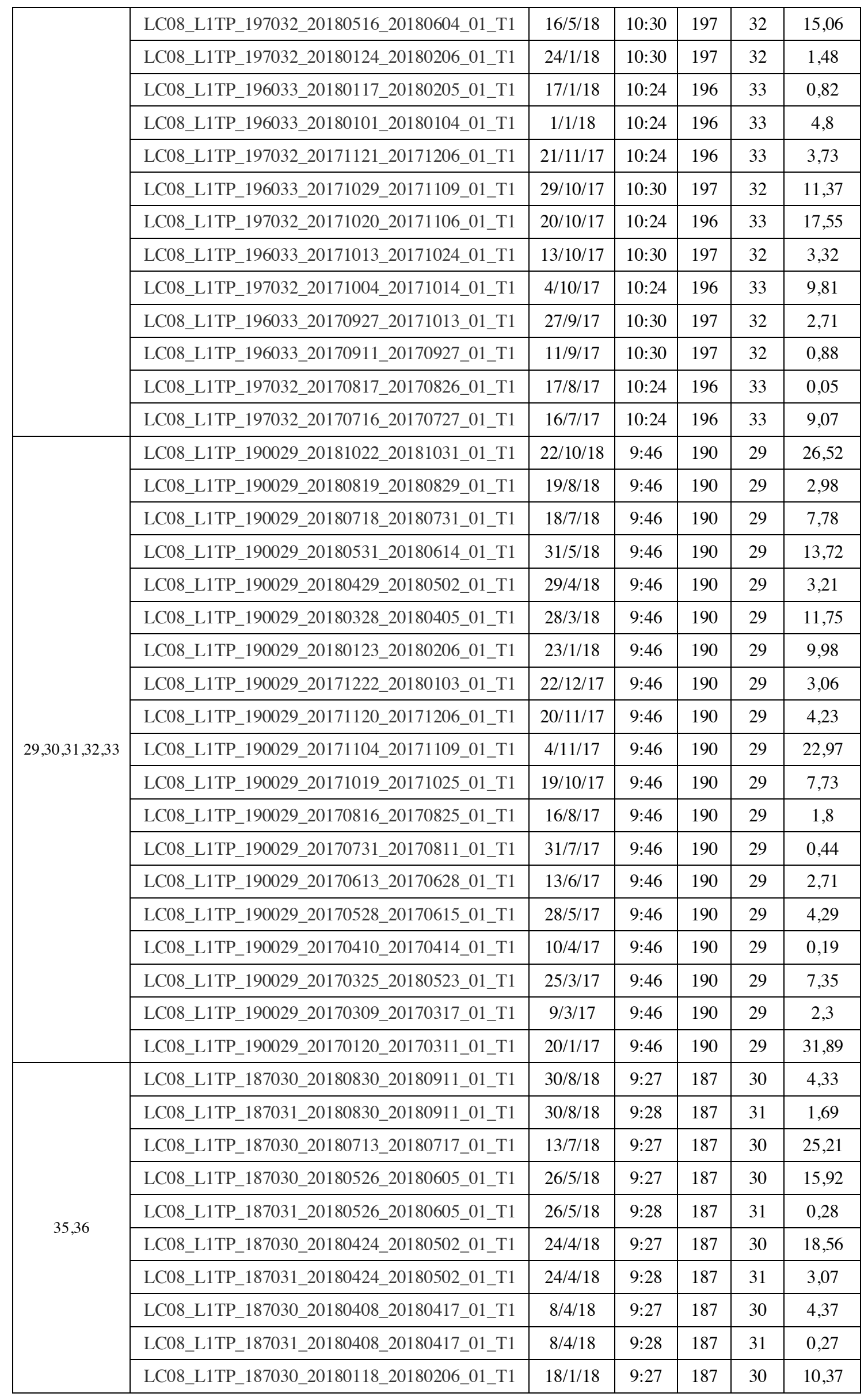




\begin{tabular}{|c|c|c|c|c|c|c|}
\hline & LC08_L1TP_187031_20180118_20180206_01_T1 & $18 / 1 / 18$ & $9: 28$ & 187 & 31 & 1,43 \\
\hline & LC08_L1TP_187030_20170827_20170914_01_T1 & $27 / 8 / 17$ & $9: 27$ & 187 & 30 & 3,6 \\
\hline & LC08_L1TP_187031_20170827_20170914_01_T1 & $27 / 8 / 17$ & $9: 28$ & 187 & 31 & 6,85 \\
\hline & LC08_L1TP_187030_20170811_20170824_01_T1 & $11 / 8 / 17$ & $9: 27$ & 187 & 30 & 0,16 \\
\hline & LC08_L1TP_187031_20170811_20170824_01_T1 & $11 / 8 / 17$ & $9: 28$ & 187 & 31 & 2,63 \\
\hline & LC08_L1TP_187030_20170710_20170725_01_T1 & $10 / 7 / 17$ & $9: 27$ & 187 & 30 & 0,17 \\
\hline & LC08_L1TP_187031_20170710_20170725_01_T1 & $10 / 7 / 17$ & $9: 28$ & 187 & 31 & 0,36 \\
\hline & LC08_L1TP_187030_20170608_20170616_01_T1 & $8 / 6 / 17$ & 9:27 & 187 & 30 & 36,01 \\
\hline & LC08_L1TP_187031_20170608_20170616_01_T1 & $8 / 6 / 17$ & $9: 28$ & 187 & 31 & 0,52 \\
\hline & LC08_L1TP_187030_20170523_20170526_01_T1 & $23 / 5 / 17$ & $9: 27$ & 187 & 30 & 30,46 \\
\hline & LC08_L1TP_187031_20170523_20170526_01_T1 & $23 / 5 / 17$ & $9: 28$ & 187 & 31 & 7,41 \\
\hline & LC08_L1TP_187030_20170304_20170316_01_T1 & $4 / 3 / 17$ & $9: 27$ & 187 & 30 & 8,83 \\
\hline & LC08_L1TP_187031_20170304_20170316_01_T1 & $4 / 3 / 17$ & $9: 28$ & 187 & 31 & 0,75 \\
\hline & LC08_L1TP_187030_20170216_20170228_01_T1 & $16 / 2 / 17$ & $9: 27$ & 187 & 30 & 0,98 \\
\hline & LC08_L1TP_187031_20170216_20170228_01_T1 & $16 / 2 / 17$ & $9: 28$ & 187 & 31 & 2,02 \\
\hline \multirow{22}{*}{34} & LC08_L1TP_188030_20181227_20190129_01_T1 & $27 / 12 / 18$ & $9: 34$ & 188 & 30 & 11,5 \\
\hline & LC08_L1TP_188030_20181109_20181116_01_T1 & $9 / 11 / 18$ & $9: 34$ & 188 & 30 & 4,66 \\
\hline & LC08_L1TP_188030_20181008_20181029_01_T1 & $8 / 10 / 18$ & $9: 34$ & 188 & 30 & 4,66 \\
\hline & LC08_L1TP_188030_20180922_20180928_01_T1 & $22 / 9 / 18$ & $9: 34$ & 188 & 30 & 7 \\
\hline & LC08_L1TP_188030_20180906_20180912_01_T1 & $6 / 9 / 18$ & $9: 34$ & 188 & 30 & 6,9 \\
\hline & LC08_L1TP_188030_20180821_20180829_01_T1 & $21 / 8 / 18$ & $9: 34$ & 188 & 30 & 2,81 \\
\hline & LC08_L1TP_188030_20180720_20180731_01_T1 & $20 / 7 / 18$ & 9:34 & 188 & 30 & 0,57 \\
\hline & LC08_L1TP_188030_20180704_20180717_01_T1 & $4 / 7 / 18$ & $9: 34$ & 188 & 30 & 20,2 \\
\hline & LC08_L1TP_188030_20180602_20180615_01_T1 & $2 / 6 / 18$ & $9: 34$ & 188 & 30 & 1,84 \\
\hline & LC08_L1TP_188030_20180125_20180206_01_T1 & $25 / 1 / 18$ & $9: 34$ & 188 & 30 & 2,3 \\
\hline & LC08_L1TP_188030_20171224_20180103_01_T1 & $24 / 12 / 17$ & $9: 34$ & 188 & 30 & 5,63 \\
\hline & LC08_L1TP_188030_20171021_20171106_01_T1 & $21 / 10 / 17$ & $9: 34$ & 188 & 30 & 5,95 \\
\hline & LC08_L1TP_188030_20171005_20171014_01_T1 & $5 / 10 / 17$ & 9:34 & 188 & 30 & 18,17 \\
\hline & LC08_L1TP_188030_20170818_20170826_01_T1 & $18 / 8 / 17$ & $9: 34$ & 188 & 30 & 0,45 \\
\hline & LC08_L1TP_188030_20170802_20170812_01_T1 & $2 / 8 / 17$ & $9: 34$ & 188 & 30 & 0,03 \\
\hline & LC08_L1TP_188030_20170717_20170727_01_T1 & $17 / 7 / 17$ & $9: 34$ & 188 & 30 & 8,62 \\
\hline & LC08_L1TP_188030_20170530_20180527_01_T1 & $30 / 5 / 17$ & $9: 34$ & 188 & 30 & 1,94 \\
\hline & LC08_L1TP_188030_20170514_20180527_01_T1 & $14 / 5 / 17$ & $9: 34$ & 188 & 30 & 9,81 \\
\hline & LC08_L1TP_188030_20170412_20180527_01_T1 & $12 / 4 / 17$ & $9: 34$ & 188 & 30 & 0,71 \\
\hline & LC08_L1TP_188030_20170327_20170414_01_T1 & $27 / 3 / 17$ & $9: 34$ & 188 & 30 & 17,8 \\
\hline & LC08_L1TP_188030_20170311_20170317_01_T1 & $11 / 3 / 17$ & $9: 34$ & 188 & 30 & 30,07 \\
\hline & LC08_L1TP_188030_20170122_20170311_01_T1 & $22 / 1 / 17$ & $9: 34$ & 188 & 30 & 6,15 \\
\hline \multirow{5}{*}{37} & LC08_L1TP_185033_20180917_20180928_01_T1 & $17 / 9 / 18$ & 9:16 & 185 & 33 & 1,29 \\
\hline & LC08_L1TP_185033_20180901_20180912_01_T1 & $1 / 9 / 18$ & $9: 16$ & 185 & 33 & 1,56 \\
\hline & LC08_L1TP_185033_20180731_20180814_01_T1 & $31 / 7 / 18$ & $9: 16$ & 185 & 33 & 4,6 \\
\hline & LC08_L1TP_185033_20180715_20180730_01_T1 & $15 / 7 / 18$ & $9: 16$ & 185 & 33 & 0,65 \\
\hline & LC08_L1TP_185033_20180613_20180615_01_T1 & $13 / 6 / 18$ & $9: 16$ & 185 & 33 & 3,6 \\
\hline
\end{tabular}




\begin{tabular}{|c|c|c|c|c|c|c|}
\hline & LC08_L1TP_185033_20180528_20180605_01_T1 & $28 / 5 / 18$ & $9: 16$ & 185 & 33 & 13,16 \\
\hline & LC08_L1TP_185033_20180512_20180517_01_T1 & $12 / 5 / 18$ & $9: 16$ & 185 & 33 & 5,64 \\
\hline & LC08_L1TP_185033_20180426_20180502_01_T1 & $26 / 4 / 18$ & $9: 16$ & 185 & 33 & 14,84 \\
\hline & LC08_L1TP_185033_20180205_20180220_01_T1 & $5 / 2 / 18$ & $9: 16$ & 185 & 33 & 1,16 \\
\hline & LC08_L1TP_185033_20171016_20171024_01_T1 & $16 / 10 / 17$ & $9: 16$ & 185 & 33 & 0,05 \\
\hline & LC08_L1TP_185033_20170914_20170928_01_T1 & $14 / 9 / 17$ & $9: 16$ & 185 & 33 & 0,06 \\
\hline & LC08_L1TP_185033_20170728_20170810_01_T1 & $28 / 7 / 17$ & $9: 16$ & 185 & 33 & 3,29 \\
\hline & LC08_L1TP_185033_20170712_20170726_01_T1 & $12 / 7 / 17$ & $9: 16$ & 185 & 33 & 1,14 \\
\hline & LC08_L1TP_185033_20170626_20180527_01_T1 & $26 / 6 / 17$ & $9: 16$ & 185 & 33 & 1,54 \\
\hline & LC08_L1TP_185033_20170610_20180527_01_T1 & $10 / 6 / 17$ & $9: 16$ & 185 & 33 & 7,04 \\
\hline & LC08_L1TP_185033_20170423_20170502_01_T1 & $23 / 4 / 17$ & $9: 16$ & 185 & 33 & 4,12 \\
\hline & LC08_L1TP_185033_20170101_20170312_01_T1 & $1 / 1 / 17$ & $9: 16$ & 185 & 33 & 1,94 \\
\hline \multirow{23}{*}{$38,39,40$} & LC08_L1TP_183034_20181224_20190129_01_T1 & $24 / 12 / 18$ & 9:04 & 183 & 34 & 1,82 \\
\hline & LC08_L1TP_183034_20181208_20181211_01_T1 & $8 / 12 / 18$ & 9:04 & 183 & 34 & 1,52 \\
\hline & LC08_L1TP_183034_20181106_20181115_01_T1 & $6 / 11 / 18$ & 9:04 & 183 & 34 & 6,35 \\
\hline & LC08_L1TP_183034_20180919_20180928_01_T1 & $19 / 9 / 18$ & 9:04 & 183 & 34 & 2 \\
\hline & LC08_L1TP_183034_20180919_20180928_01_T1 & $3 / 9 / 18$ & 9:04 & 183 & 34 & 0,02 \\
\hline & LC08_L1TP_183034_20180818_20180829_01_T1 & $18 / 8 / 18$ & 9:04 & 183 & 34 & 1,9 \\
\hline & LC08_L1TP_183034_20180802_20180814_01_T1 & $2 / 8 / 18$ & 9:0 & 183 & 34 & 10,41 \\
\hline & LC08_L1TP_183034_20180717_20180730_01_T1 & $17 / 7 / 18$ & 9:04 & 183 & 34 & 0,16 \\
\hline & LC08_L1TP_183034_20180530_20180614_01_T1 & $30 / 5 / 18$ & 9:04 & 183 & 34 & 6,55 \\
\hline & LC08_L1TP_183034_20180514_20180517_01_T1 & $14 / 5 / 18$ & 9:04 & 183 & 34 & 1,27 \\
\hline & LC08_L1TP_183034_20180428_20180502_01_T1 & $28 / 4 / 18$ & 9:04 & 183 & 34 & 0,09 \\
\hline & LC08_L1TP_183034_20180106_20180119_01_T1 & $6 / 1 / 18$ & 9:04 & 183 & 34 & 1,63 \\
\hline & LC08_L1TP_183034_20171205_20171222_01_T1 & $5 / 12 / 17$ & 9:04 & 183 & 34 & 5,74 \\
\hline & LC08_L1TP_183034_20170916_20170929_01_T1 & $16 / 9 / 17$ & 9:04 & 183 & 34 & 0,02 \\
\hline & LC08_L1TP_183034_20170831_20170915_01_T1 & $31 / 8 / 17$ & 9:04 & 183 & 34 & 2,68 \\
\hline & LC08_L1TP_183034_20170815_20170825_01_T1 & $15 / 8 / 17$ & 9:04 & 183 & 34 & 5,23 \\
\hline & LC08_L1TP_183034_20170730_20170811_01_T1 & $30 / 7 / 17$ & 9:04 & 183 & 34 & 0,04 \\
\hline & LC08_L1TP_183034_20170714_20170726_01_T1 & $14 / 7 / 17$ & 9:04 & 183 & 34 & 1,9 \\
\hline & LC08_L1TP_183034_20170628_20170714_01_T1 & $28 / 6 / 17$ & 9:04 & 183 & 34 & 2,94 \\
\hline & LC08_L1TP_183034_20170511_20170516_01_T1 & $11 / 5 / 17$ & 9:04 & 183 & 34 & 1,24 \\
\hline & LC08_L1TP_183034_20170425_20170502_01_T1 & $25 / 4 / 17$ & 9:04 & 183 & 34 & 2,5 \\
\hline & LC08_L1TP_183034_20170324_20180527_01_T1 & $24 / 3 / 17$ & 9:04 & 183 & 34 & 0,21 \\
\hline & LC08_L1TP_183034_20170103_20170312_01_T1 & $3 / 1 / 17$ & 9:04 & 183 & 34 & 1,83 \\
\hline \multirow{7}{*}{41,42} & LC08_L1TP_181036_20181124_20181210_01_T1 & $8 / 11 / 18$ & $8: 53$ & 181 & 36 & 17,46 \\
\hline & LC08_L1TP_181036_20181007_20181029_01_T1 & $7 / 10 / 18$ & $8: 53$ & 181 & 36 & 5,15 \\
\hline & LC08_L1TP_181036_20180921_20180928_01_T1 & $21 / 9 / 18$ & $8: 53$ & 181 & 36 & 3,12 \\
\hline & LC08_L1TP_181036_20180905_20180912_01_T1 & $5 / 9 / 18$ & $8: 53$ & 181 & 36 & 0,03 \\
\hline & LC08_L1TP_181036_20180820_20180829_01_T1 & $20 / 8 / 18$ & $8: 53$ & 181 & 36 & 1,24 \\
\hline & LC08_L1TP_181036_20180719_20180731_01_T1 & $19 / 7 / 18$ & $8: 53$ & 181 & 36 & 0,15 \\
\hline & LC08_L1TP_181036_20180703_20180717_01_T1 & $3 / 7 / 18$ & $8: 53$ & 181 & 36 & 0,7 \\
\hline
\end{tabular}




\begin{tabular}{|c|c|c|c|c|c|c|}
\hline & LC08_L1TP_181036_20180601_20180614_01_T1 & $1 / 6 / 18$ & $8: 53$ & 181 & 36 & 0,05 \\
\hline & LC08_L1TP_181036_20180516_20180604_01_T1 & $16 / 5 / 18$ & $8: 53$ & 181 & 36 & 5,94 \\
\hline & LC08_L1TP_181036_20180430_20180502_01_T1 & $30 / 4 / 18$ & $8: 53$ & 181 & 36 & 6,22 \\
\hline & LC08_L1TP_181036_20180414_20180501_01_T1 & $14 / 4 / 18$ & $8: 53$ & 181 & 36 & 15,35 \\
\hline & LC08_L1TP_181036_20180225_20180308_01_T & $25 / 2 / 18$ & $8: 53$ & 181 & 36 & 42,38 \\
\hline & LC08_L1TP_181036_20180108_20180119_01_T1 & $8 / 1 / 18$ & $8: 53$ & 181 & 36 & 2,55 \\
\hline & LC08_L1TP_181036_20171020_20171106_01_T1 & $20 / 10 / 17$ & $8: 53$ & 181 & 36 & 5,75 \\
\hline & LC08_L1TP_181036_20171004_20171014_01_T1 & $4 / 10 / 17$ & $8: 53$ & 181 & 36 & 1,28 \\
\hline & LC08_L1TP_181036_20170918_20180527_01_T1 & $18 / 9 / 17$ & $8: 53$ & 181 & 36 & 0 \\
\hline & LC08_L1TP_181036_20170902_20170916_01_T1 & $2 / 9 / 17$ & $8: 53$ & 181 & 36 & 0 \\
\hline & LC08_L1TP_181036_20170801_20170811_01_T1 & $1 / 8 / 17$ & $8: 53$ & 181 & 36 & 0,78 \\
\hline & LC08_L1TP_181036_20170716_20170727_01_T1 & $16 / 7 / 17$ & $8: 53$ & 181 & 36 & 2,43 \\
\hline \multirow{18}{*}{$43,44,45$} & LC08_L1TP_180034_20181101_20181115_01_T1 & $1 / 11 / 18$ & $8: 46$ & 180 & 34 & 0.07 \\
\hline & LC08_L1TP_180034_20181016_20181030_01_T1 & $16 / 10 / 18$ & $8: 46$ & 180 & 34 & 0 \\
\hline & LC08_L1TP_180034_20180914_20180928_01_T1 & $14 / 9 / 18$ & $8: 46$ & 180 & 34 & 5.57 \\
\hline & LC08_L1TP_180034_20180813_20180828_01_T1 & $13 / 8 / 18$ & $8: 46$ & 180 & 34 & 3.31 \\
\hline & LC08_L1TP_180034_20180728_20180731_01_T1 & $28 / 7 / 18$ & $8: 46$ & 180 & 34 & 2.09 \\
\hline & LC08_L1TP_180034_20180712_20180717_01_T1 & $12 / 7 / 18$ & $8: 46$ & 180 & 34 & 0.31 \\
\hline & LC08_L1TP_180034_20180610_20180615_01_T1 & $10 / 6 / 18$ & $8: 46$ & 180 & 34 & 1.52 \\
\hline & LC08_L1TP_180034_20180509_20180517_01_T1 & $9 / 5 / 18$ & $8: 46$ & 180 & 34 & 11.93 \\
\hline & LC08_L1TP_180034_20180423_20180502_01_T1 & $23 / 4 / 18$ & $8: 46$ & 180 & 34 & 5 \\
\hline & LC08_L1TP_180034_20180218_20180307_01_T1 & $18 / 2 / 18$ & $8: 46$ & 180 & 34 & 0.65 \\
\hline & LC08_L1TP_180034_20180101_20180104_01_T1 & $1 / 1 / 18$ & $8: 46$ & 180 & 34 & 1.19 \\
\hline & LC08_L1TP_180034_20171013_20171024_01_T1 & $13 / 10 / 17$ & $8: 46$ & 180 & 34 & 0.04 \\
\hline & LC08_L1TP_180034_20170927_20171013_01_T1 & $27 / 9 / 17$ & $8: 46$ & 180 & 34 & 2.01 \\
\hline & LC08_L1TP_180034_20170826_20170913_01_T1 & $26 / 8 / 17$ & $8: 46$ & 180 & 34 & 0.02 \\
\hline & LC08_L1TP_180034_20170709_20170717_01_T1 & $9 / 7 / 17$ & $8: 46$ & 180 & 34 & 0.05 \\
\hline & LC08_L1TP_180034_20170506_20170515_01_T1 & $6 / 5 / 17$ & $8: 46$ & 180 & 34 & 10.42 \\
\hline & LC08_L1TP_180034_20170303_20170316_01_T1 & $3 / 3 / 17$ & $8: 46$ & 180 & 34 & 9.52 \\
\hline & LC08_L1TP_180034_20170215_20170228_01_T1 & $15 / 2 / 17$ & $8: 46$ & 180 & 34 & 25.38 \\
\hline \multirow{10}{*}{$46,47,48$} & LC08_L1TP_178034_20181221_20181227_01_T1 & $21 / 12 / 18$ & $8: 33$ & 178 & 34 & 17.22 \\
\hline & LC08_L1TP_178034_20181103_20181115_01_T1 & $3 / 11 / 18$ & $8: 33$ & 178 & 34 & 0.07 \\
\hline & LC08_L1TP_178034_20180916_20180928_01_T1 & $16 / 9 / 18$ & $8: 33$ & 178 & 34 & 0.07 \\
\hline & LC08_L1TP_178034_20180815_20180828_01_T1 & $15 / 8 / 18$ & $8: 33$ & 178 & 34 & 0.04 \\
\hline & LC08_L1TP_178034_20180119_20180206_01_T1 & $19 / 1 / 18$ & $8: 33$ & 178 & 34 & 8.56 \\
\hline & LC08_L1TP_178034_20171031_20171109_01_T1 & $31 / 10 / 17$ & $8: 33$ & 178 & 34 & 4.76 \\
\hline & LC08_L1TP_178034_20170929_20171013_01_T1 & $29 / 9 / 17$ & $8: 33$ & 178 & 34 & 15.51 \\
\hline & LC08_L1TP_178034_20170828_20170914_01_T1 & 28/8/17 & $8: 33$ & 178 & 34 & 0.15 \\
\hline & LC08_L1TP_178034_20170321_20170328_01_T1 & $21 / 3 / 17$ & $8: 33$ & 178 & 34 & 21.56 \\
\hline & LC08_L1TP_178034_20170217_20170228_01_T1 & $17 / 2 / 17$ & $8: 33$ & 178 & 34 & 1.69 \\
\hline \multirow{2}{*}{49} & LC08_L1TP_176036_20181105_20181115_01_T1 & $5 / 11 / 18$ & $8: 22$ & 176 & 36 & 27.70 \\
\hline & LC08_L1TP_176036_20181004_20181010_01_T1 & $4 / 10 / 18$ & $8: 22$ & 176 & 36 & 3.48 \\
\hline
\end{tabular}




\begin{tabular}{|c|c|c|c|c|c|c|}
\hline & LC08_L1TP_176036_20180918_20180928_01_T1 & $18 / 9 / 18$ & $8: 22$ & 176 & 36 & 0.17 \\
\hline & LC08_L1TP_176036_20180326_20180404_01_T1 & $26 / 3 / 18$ & $8: 22$ & 176 & 36 & 1.03 \\
\hline & LC08_L1TP_176036_20180206_20180221_01_T1 & $6 / 2 / 18$ & $8: 22$ & 176 & 36 & 3.11 \\
\hline & LC08_L1TP_176036_20171017_20171024_01_T1 & $17 / 10 / 17$ & $8: 22$ & 176 & 36 & 0.60 \\
\hline & LC08_L1TP_176036_20170915_20180526_01_T1 & $15 / 9 / 17$ & $8: 22$ & 176 & 36 & 5.60 \\
\hline & LC08_L1TP_176036_20170830_20170914_01_T1 & $30 / 8 / 17$ & $8: 22$ & 176 & 36 & 1.20 \\
\hline & LC08_L1TP_176036_20170729_20170811_01_T1 & $29 / 7 / 17$ & $8: 22$ & 176 & 36 & 2.78 \\
\hline & LC08_L1TP_176036_20170510_20170516_01_T1 & $10 / 5 / 17$ & $8: 22$ & 176 & 36 & 0.02 \\
\hline & LC08_L1TP_176036_20170424_20170502_01_T1 & $24 / 4 / 17$ & $8: 22$ & 176 & 36 & 0.18 \\
\hline & LC08_L1TP_176036_20170307_20170317_01_T1 & $7 / 3 / 16$ & $8: 22$ & 176 & 36 & 0.49 \\
\hline & LC08_L1TP_176036_20170118_20170311_01_T1 & $18 / 1 / 17$ & $8: 22$ & 176 & 36 & 0.75 \\
\hline \multirow{13}{*}{50} & LC08_L1TP_176035_20181004_20181010_01_T1 & $4 / 10 / 18$ & $8: 21$ & 176 & 35 & 0.18 \\
\hline & LC08_L1TP_176035_20180918_20180928_01_T1 & $18 / 9 / 18$ & $8: 21$ & 176 & 35 & 1.71 \\
\hline & LC08_L1TP_176035_20180817_20180829_01_T1 & $17 / 8 / 18$ & $8: 21$ & 176 & 35 & 5.32 \\
\hline & LC08_L1TP_176035_20180716_20180730_01_T1 & $16 / 7 / 18$ & $8: 21$ & 176 & 35 & 2.62 \\
\hline & LC08_L1TP_176035_20180630_20180716_01_T1 & $30 / 6 / 18$ & $8: 21$ & 176 & 35 & 6.85 \\
\hline & LC08_L1TP_176035_20180326_20180404_01_T1 & $26 / 3 / 18$ & $8: 21$ & 176 & 35 & 2.19 \\
\hline & LC08_L1TP_176035_20180206_20180221_01_T1 & $6 / 2 / 18$ & $8: 21$ & 176 & 35 & 1.04 \\
\hline & LC08_L1TP_176035_20171017_20171024_01_T1 & $17 / 10 / 17$ & $8: 21$ & 176 & 35 & 0.25 \\
\hline & LC08_L1TP_176035_20170729_20170811_01_T1 & $29 / 7 / 17$ & $8: 21$ & 176 & 35 & 0.77 \\
\hline & LC08_L1TP_176035_20170526_20170616_01_T1 & $26 / 5 / 17$ & $8: 21$ & 176 & 35 & 3.31 \\
\hline & LC08_L1TP_176035_20170424_20170502_01_T1 & $24 / 4 / 17$ & $8: 21$ & 176 & 35 & 0.78 \\
\hline & LC08_L1TP_176035_20170323_20170329_01_T1 & $23 / 3 / 17$ & $8: 21$ & 176 & 35 & 4.96 \\
\hline & LC08_L1TP_176035_20170118_20170311_01_T1 & $18 / 1 / 17$ & $8: 21$ & 176 & 35 & 0.87 \\
\hline \multirow{14}{*}{51,52} & LC08_L1TP_174036_20181107_20181116_01_T1 & $7 / 11 / 18$ & 8:09 & 174 & 36 & 17.03 \\
\hline & LC08_L1TP_174036_20180702_20180716_01_T1 & $7 / 11 / 18$ & 8:09 & 174 & 36 & 2.37 \\
\hline & LC08_L1TP_174036_20180515_20180604_01_T1 & $7 / 11 / 18$ & 8:09 & 174 & 36 & 2.53 \\
\hline & LC08_L1TP_174036_20180413_20180417_01_T1 & $7 / 11 / 18$ & 8:09 & 174 & 36 & 1.49 \\
\hline & LC08_L1TP_174036_20180224_20180308_01_T1 & $7 / 11 / 18$ & 8:09 & 174 & 36 & 12.59 \\
\hline & LC08_L1TP_174036_20180107_20180119_01_T1 & $7 / 11 / 18$ & 8:09 & 174 & 36 & 1.57 \\
\hline & LC08_L1TP_174036_20171019_20171025_01_T1 & $7 / 11 / 18$ & 8:09 & 174 & 36 & 0.02 \\
\hline & LC08_L1TP_174036_20171003_20171014_01_T1 & $7 / 11 / 18$ & 8:09 & 174 & 36 & 1.94 \\
\hline & LC08_L1TP_174036_20170901_20170915_01_T1 & $7 / 11 / 18$ & 8:09 & 174 & 36 & 5.97 \\
\hline & LC08_L1TP_174036_20170731_20170811_01_T1 & $7 / 11 / 18$ & 8:09 & 174 & 36 & 1.46 \\
\hline & LC08_L1TP_174036_20170528_20170615_01_T1 & $7 / 11 / 18$ & 8:09 & 174 & 36 & 1.25 \\
\hline & LC08_L1TP_174036_20170512_20170525_01_T1 & $7 / 11 / 18$ & 8:09 & 174 & 36 & 1.07 \\
\hline & LC08_L1TP_174036_20170325_20170329_01_T1 & $7 / 11 / 18$ & 8:09 & 174 & 36 & 1.50 \\
\hline & LC08_L1TP_174036_20170221_20170301_01_T1 & $7 / 11 / 18$ & 8:09 & 174 & 36 & 1.85 \\
\hline \multirow{4}{*}{53} & LC08_L1TP_174037_20181225_20190129_01_T1 & $25 / 12 / 18$ & $8: 10$ & 174 & 37 & 29.85 \\
\hline & LC08_L1TP_174037_20181107_20181116_01_T1 & $7 / 11 / 18$ & $8: 10$ & 174 & 37 & 16.73 \\
\hline & LC08_L1TP_174037_20180920_20180928_01_T1 & $20 / 9 / 18$ & $8: 10$ & 174 & 37 & 1.10 \\
\hline & LC08_L1TP_174037_20180904_20180912_01_T1 & $4 / 9 / 18$ & $8: 10$ & 174 & 37 & 0.88 \\
\hline
\end{tabular}




\begin{tabular}{|c|c|c|c|c|c|c|}
\hline & LC08_L1TP_174037_20180702_20180716_01_T1 & $2 / 7 / 18$ & $8: 10$ & 174 & 37 & 0.91 \\
\hline & LC08_L1TP_174037_20180616_20180703_01_T1 & $16 / 6 / 18$ & $8: 10$ & 174 & 37 & 0.02 \\
\hline & LC08_L1TP_174037_20180413_20180417_01_T1 & $13 / 4 / 18$ & $8: 10$ & 174 & 37 & 0.23 \\
\hline & LC08_L1TP_174037_20180224_20180308_01_T1 & $24 / 2 / 18$ & $8: 10$ & 174 & 37 & 7.47 \\
\hline & LC08_L1TP_174037_20180107_20180119_01_T1 & $7 / 1 / 18$ & $8: 10$ & 174 & 37 & 2.47 \\
\hline & LC08_L1TP_174037_20171019_20171025_01_T1 & $19 / 10 / 17$ & $8: 10$ & 174 & 37 & 0.06 \\
\hline & LC08_L1TP_174037_20170731_20170811_01_T1 & $31 / 7 / 17$ & $8: 10$ & 174 & 37 & 0.84 \\
\hline & LC08_L1TP_174037_20170715_20170727_01_T1 & $15 / 7 / 17$ & $8: 10$ & 174 & 37 & 0.29 \\
\hline & LC08_L1TP_174037_20170528_20170615_01_T1 & $28 / 5 / 17$ & $8: 10$ & 174 & 37 & 0.51 \\
\hline & LC08_L1TP_174037_20170512_20170525_01_T1 & $12 / 5 / 17$ & $8: 10$ & 174 & 37 & 0.25 \\
\hline & LC08_L1TP_174037_20170325_20170329_01_T1 & $25 / 3 / 17$ & $8: 10$ & 174 & 37 & 2.85 \\
\hline & LC08_L1TP_174037_20170221_20170301_01_T1 & $21 / 2 / 17$ & $8: 10$ & 174 & 37 & 1.31 \\
\hline & LC08_L1TP_174037_20170205_20170216_01_T1 & $5 / 2 / 17$ & $8: 10$ & 174 & 37 & 1.80 \\
\hline & LC08_L1TP_174037_20170120_20170311_01_T1 & 20/1/17 & $8: 10$ & 174 & 37 & 13.20 \\
\hline \multirow{24}{*}{54} & LC08_L1TP_184038_20181012_20181030_01_T1 & $12 / 10 / 18$ & $9: 12$ & 184 & 38 & 17,32 \\
\hline & LC08_L1TP_184038_20180910_20180913_01_T1 & $10 / 9 / 18$ & $9: 12$ & 184 & 38 & 7,37 \\
\hline & LC08_L1TP_184038_20180809_20180815_01_T1 & $9 / 8 / 18$ & $9: 12$ & 184 & 38 & 0 \\
\hline & LC08_L1TP_184038_20180708_20180717_01_T1 & $8 / 7 / 18$ & $9: 12$ & 184 & 38 & 0,17 \\
\hline & LC08_L1TP_184038_20180622_20180703_01_T1 & $11 / 6 / 18$ & $9: 12$ & 184 & 38 & 12,24 \\
\hline & LC08_L1TP_184038_20180606_20180615_01_T1 & $6 / 6 / 18$ & $9: 12$ & 184 & 38 & 0,01 \\
\hline & LC08_L1TP_184038_20180505_20180517_01_T1 & $5 / 5 / 18$ & $9: 12$ & 184 & 38 & 8,93 \\
\hline & LC08_L1TP_184038_20180419_20180501_01_T1 & $19 / 4 / 18$ & $9: 12$ & 184 & 38 & 18,29 \\
\hline & LC08_L1TP_184038_20180403_20180417_01_T1 & $3 / 4 / 18$ & $9: 12$ & 184 & 38 & 0,12 \\
\hline & LC08_L1TP_184038_20180318_20180403_01_T1 & $18 / 3 / 18$ & $9: 12$ & 184 & 38 & 5,08 \\
\hline & LC08_L1TP_184038_20180214_20180222_01_T1 & $14 / 2 / 18$ & $9: 12$ & 184 & 38 & 5,47 \\
\hline & LC08_L1TP_184038_20171212_20171223_01_T1 & $12 / 12 / 17$ & $9: 12$ & 184 & 38 & 9,72 \\
\hline & LC08_L1TP_184038_20171126_20171206_01_T1 & $26 / 11 / 17$ & $9: 12$ & 184 & 38 & 0,36 \\
\hline & LC08_L1TP_184038_20171110_20171121_01_T1 & $10 / 11 / 17$ & $9: 12$ & 184 & 38 & 1,55 \\
\hline & LC08_L1TP_184038_20170923_20171013_01_T1 & $23 / 9 / 17$ & $9: 12$ & 184 & 38 & 0,35 \\
\hline & LC08_L1TP_184038_20170907_20170926_01_T1 & $7 / 9 / 17$ & $9: 12$ & 184 & 38 & 0,41 \\
\hline & LC08_L1TP_184038_20170822_20170911_01_T1 & $22 / 8 / 17$ & 9:12 & 184 & 38 & 9,4 \\
\hline & LC08_L1TP_184038_20170806_20170813_01_T1 & $6 / 8 / 17$ & $9: 12$ & 184 & 38 & 0,19 \\
\hline & LC08_L1TP_184038_20170721_20170728_01_T1 & $21 / 7 / 17$ & $9: 12$ & 184 & 38 & 1,74 \\
\hline & LC08_L1TP_184038_20170705_20170716_01_T1 & $5 / 7 / 17$ & $9: 12$ & 184 & 38 & 8,46 \\
\hline & LC08_L1TP_184038_20170619_20170629_01_T1 & $19 / 6 / 17$ & $9: 12$ & 184 & 38 & 16,92 \\
\hline & LC08_L1TP_184038_20170518_20170525_01_T1 & $18 / 5 / 17$ & $9: 12$ & 184 & 38 & 13,58 \\
\hline & LC08_L1TP_184038_20170416_20170501_01_T1 & $16 / 4 / 17$ & $9: 12$ & 184 & 38 & 0,01 \\
\hline & LC08_L1TP_184038_20170315_20170328_01_T1 & $15 / 3 / 17$ & $9: 12$ & 184 & 38 & 0,85 \\
\hline
\end{tabular}


Supplementary material 3
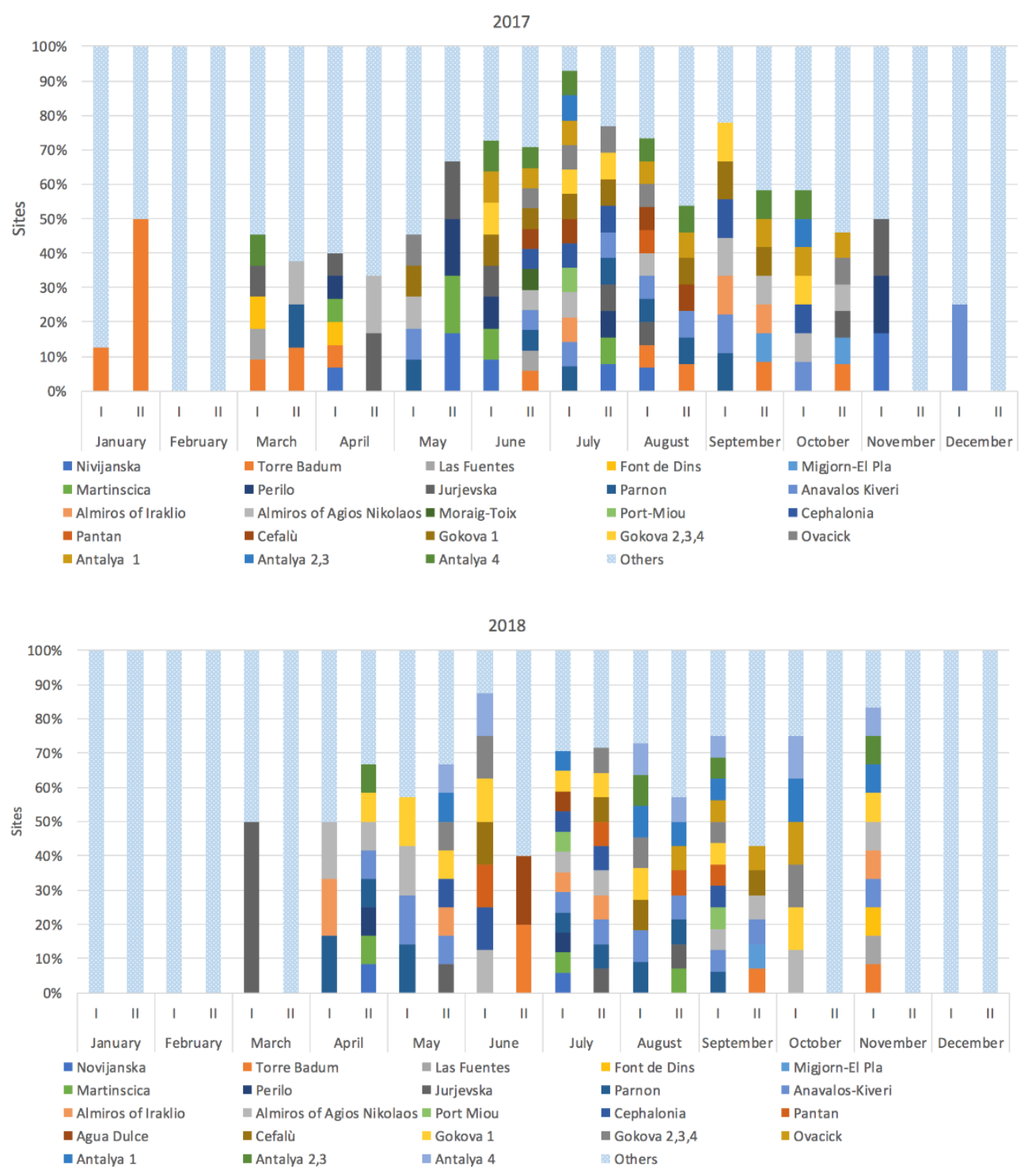
Supplementary material 4
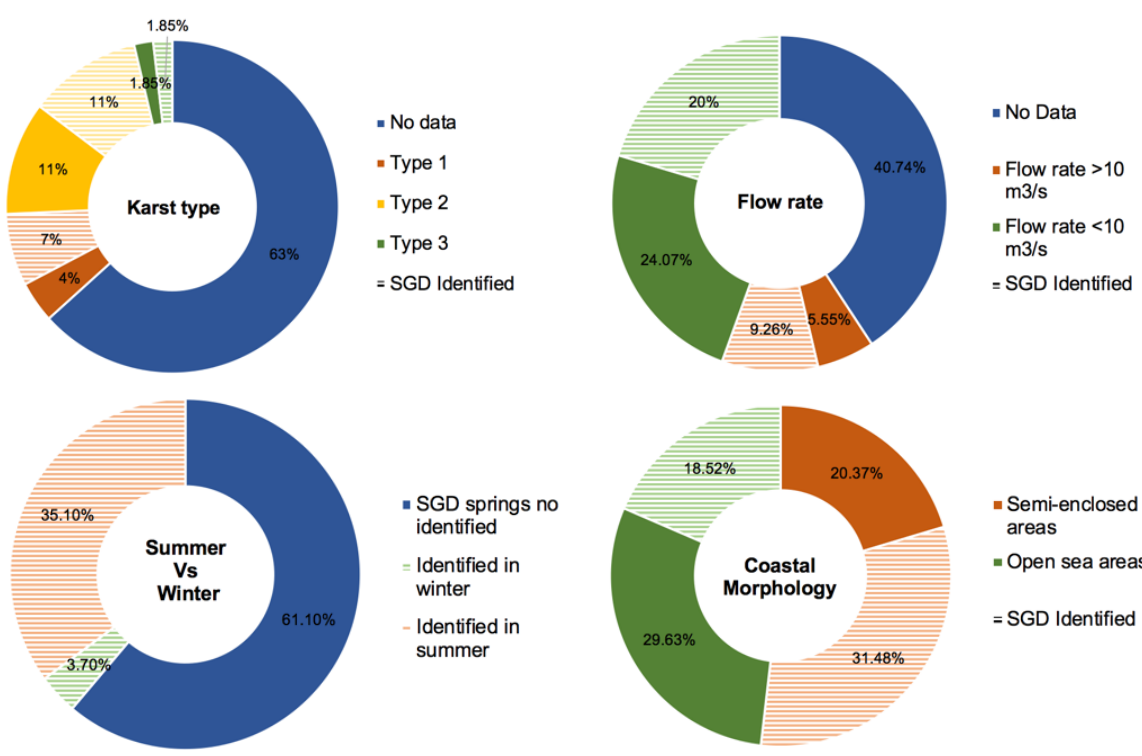

- SGD springs no identified

Identified in winter

Identified in summer
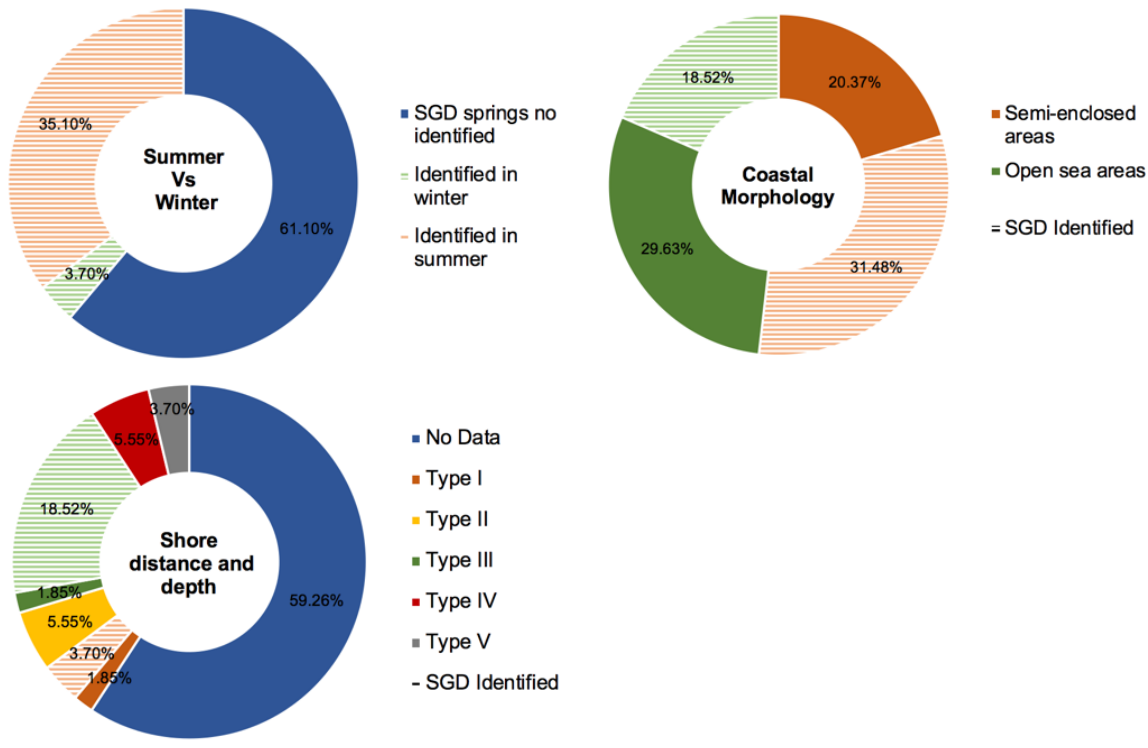

- No Data

- Type I

= Type II

- Type III

- Type IV

- Type V

- SGD Identified 


\section{Supplementary material 5}
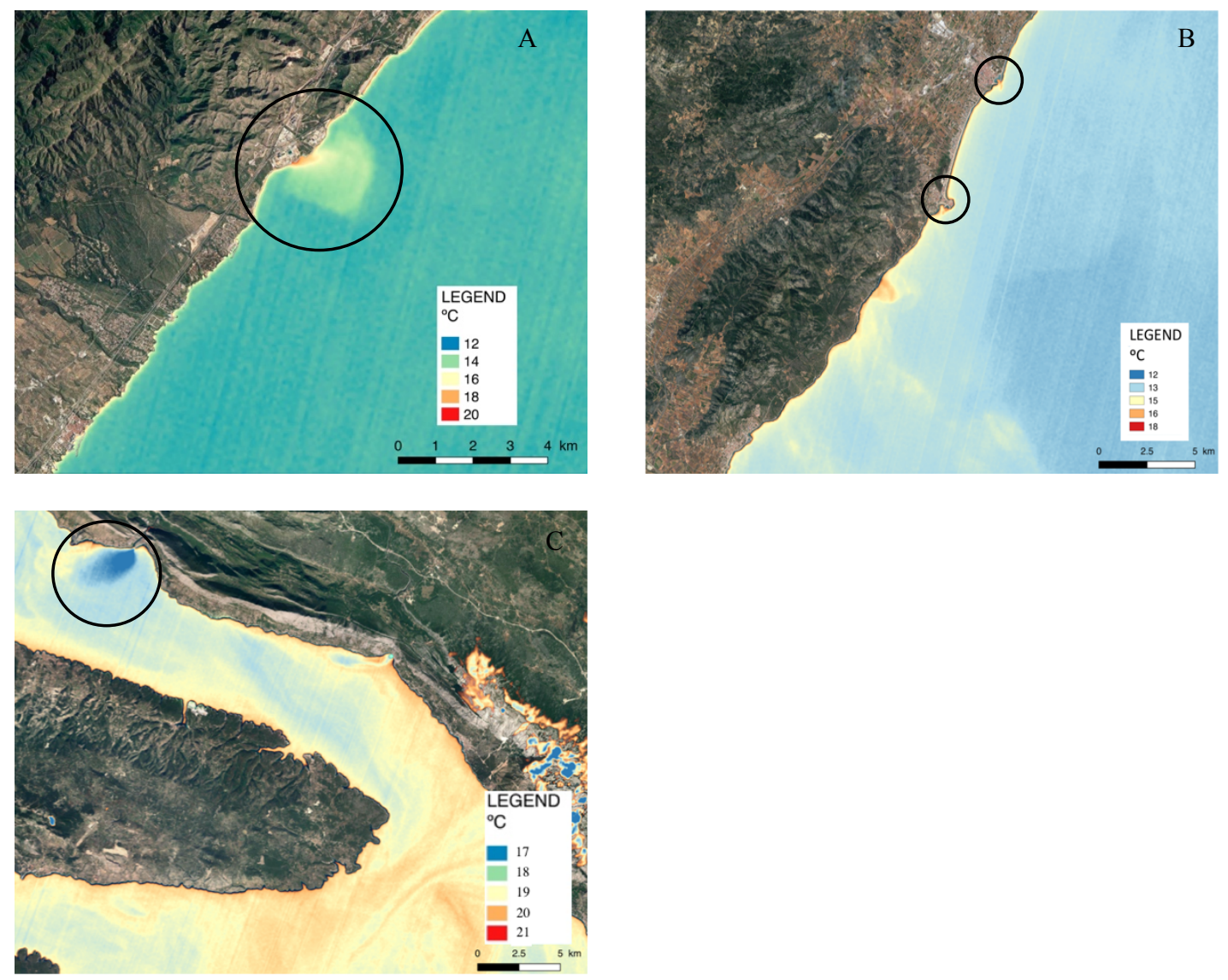

Figure: A) Vandellós II nuclear power plant in Spain, B) Benicarló port in the north and Peñiscola port in the south, both in Spain and C) Small river in Croatia. (C) Google Earth 2021. 


\section{Supplementary material 6}

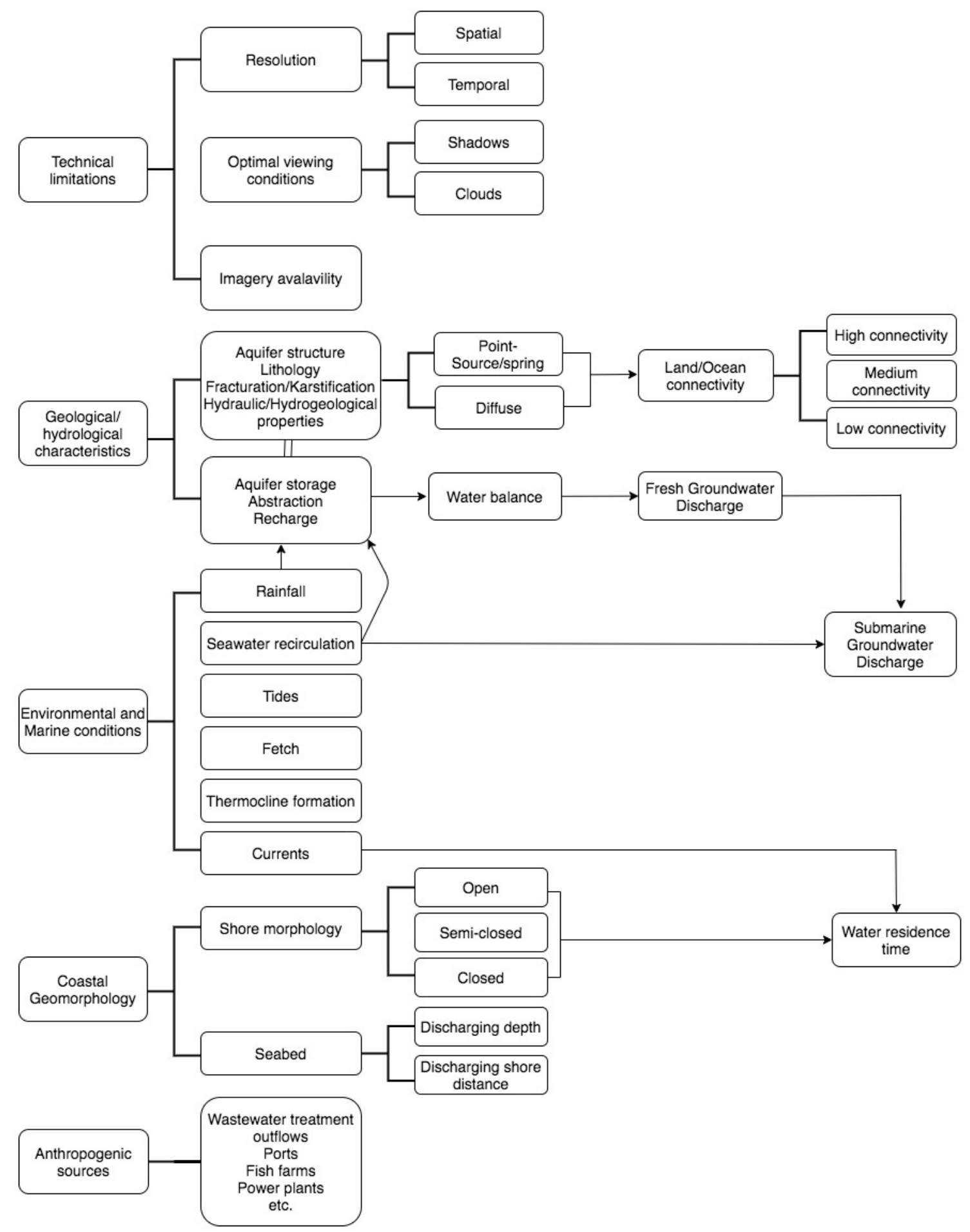

